\title{
Preliminary Development of Two Predictive Models for DNR Patients in Intensive Care
}

\author{
Mary Beth Tittle, Linda Moody, Mark P. Becker
}

The purpose of this study was to identify which variables are the best predictors of a do-not-resuscitate (DNR) classification and develop a model to predict the nursing care required by $D N R$ patients in the ICU. Data collected on DNR and non-DNR patients included nursing care requirements, severity of illness, resource allocation and sociodemographic characteristics. One model identified the best predictors of a DNR classification in intensive care as the origin of admission and the severity of illness score on the day of admission to intensive care. The second model identified the best predictors of nursing care requirements for DNR patients in intensive care as the number of days spent in intensive care prior to the DNR order, the average daily resource allocation points after the DNR order, and the severity of illness score on the day the DNR order was designated.

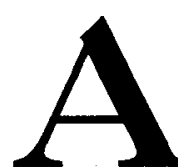

dvances in health care, including nursing care, have made it possible to greatly extend life. One such advance has been cardiopulmonary resuscitation (CPR) introduced by Kouwenhoven, Jude, and Knickerbocker (1960). Cardiopulmonary resuscitation is administered to patients who have a cardiac or respiratory arrest unless there is an order "not to resuscitate." More and more, patients are choosing not to be resuscitated and a DNR (do-not-resuscitate) order is written on the patient's medical record.

A dilemma is created when a patient with DNR orders is in the intensive care unit (ICU). The purpose of intensive care is to preserve vital life functioning and return the patient to a maximal state of health. $\AA$ decision not to resuscitate indicates that the functions of circulation and respiration will not be preserved should they fail. However, the DNR order does not preclude the use of all advanced therapies. The increasing incidence of patients with DNR orders in the ICU utilizing all advanced therapies raises important issues concerning the use of nursing and medical resources as well as the overall cost of intensive care.

Research has shown that often the most seriously ill patients admitted to the ICU have poor outcomes and use a large share of the medical and nursing resources (Cullen, Keene, Waterhaux et al., 1984; Lewandowski, Daly, McClish, Juknialis, \& Younger, 1985; Zimmerman, Knaus, Sharpe et al., 1986). Patients with DNR orders in the ICU have been shown to be such a group (Zimmerman et al., 1986). As nursing and medical resources become more scarce, the use of such resources for patients most likely to benefit becomes more important. Decisions related to DNR status and the use of nursing and medical resources need to be made based on objective data in combination with ethical values. The development of models will assist health care professionals in obtaining objective information to make these decisions.

The purpose of this study was: (a) to identify which variables are the best predictors of a DNR classification; and (b) develop a model to predict the nursing care required by DNR patients in the ICU. Two research questions were addressed:

1. Which sociodemographic and physiologic variables are the best predictors of a DNR classification of patients in intensive care?

Mary Beth Tittle, R.N., Ph.D., Delta Beta is Assistant Professor, Linda Moody. R.N., C., Ph.D., F.A.A.N. Is Professor and Director of Research, both at the college of Nursing. University of South Florida; Mark P. Becker, Ph.D. Is Assistant Professor, Department of Biostatistics, University of Michigan. This study was funded by a National Research Service Award and the Florida Nurses Foundation. Gratitude is expressed to the Medicus Systems Corporation for the use of the Nursing Productivity and Quality System in the study. Prof. Tittle also thanks Drs. Molly Dougherty, W.J. Taylor and James Wattenbarger as members of her disserta. tion research committee. Correspondence to Prof. Tittle, College of Nursing University of South Florlda, 129018 ruce 8. Downs Blvd., Box 22 Health Sciences Center, Tampa FL 33612. Accepted for publication January 23, 1991. 
2. Which variables are the best predictors of the nursing care required by DNR patients in intensive care?

\section{Review of Literature}

Intensive care units provide intensive nursing and medical care to a specialized group of patients and are an integral part of hospital functioning. Research has shown that two types of patients are admitted to the ICU: those who require intensive treatment due to a critical condition; and those who require close monitoring to prevent a life-threatening complication (Knaus, Wagner, Draper, Lawrence, \& Zimmerman, 1981; Thibault, Mulley, Barnett et al., 1981; Wagner, Knaus, Draper, \& Zimmerman, 1983). Results of these studies indicated that a large portion of admissions were for monitoring of complications.

Intensive care costs more in labor and equipment than general floor care (Draper, 1983). Research has shown an inverse relationship between cost and survival (Cullen et al., 1984; Jackson, 1984; Parno, Teres, Lemeshow, \& Brown, 1982). Some patients respond rapidly to treatment with the use of a small amount of intensive care resources, yet a small proportion of patients with poor outcomes accounts for a disproportionate amount of intensive care resources. $\mathrm{Pa}$ tients with DNR orders in intensive care are such a group (Zimmerman et al., 1986).

Little research has been conducted on the patient with a DNR order, especially in the ICU. Witte (1984) reviewed 100 medical records retrospectively to determine which of a set of specific demographic, physiologic, and psychosocial variables were present when intensive care patients were to be resuscitated or not resuscitated. The sample included the charts of 30 patients who died after a DNR order was written and 70 patients who died immediately after resuscitation. Using chi-square analysis, only the variables of length of hospital stay, level of consciousness and the documentation of wishes of family or significant others were significantly different between the two groups.

Zimmerman et al. (1986) studied patients with DNR orders in the intensive care units of 13 hospitals during two data collection periods. Data collected included age, previous health status, diagnosis, indication for ICU admission, severity of illness and type and amount of therapy. There were 7,265 ICU admissions studies, 393 of whom had a DNR order. Regression analysis indicated that significant predictors of having a DNR order were: over 65 years of age; poor chronic health status; a diagnosis of intracranial hemorrhage; admission following emergency surgery or as a medical patient; and high initial severity of illness.

The characteristics of patients with a DNR order in intensive care and the type of medical care they received was studied by Younger, Lewandowski, McClish et al. (1985). The sample consisted of 506 patients from the medical ICU, 71 of whom had a DNR order. Demographic information, severity of illness, resource allocation, prior health status, reason for intensive care admission and six-month outcome were collected for each patient through a review of medical records and interviews with health team members. Patients with DNR orders were older, more likely to be Caucasian and likely to have private physicians. Regression analysis of the sample indicated predictors of a DNR status included severity of illness, age and prior health status.

Only one study has examined nursing resources used by the patient classified as DNR. Lewandowski et al. (1985) studied the use of medical and nursing resources by DNR patients in the intensive care unit. The data for this study were obtained from a larger prospective project of 506 patients, including 71 (14 percent) who had DNR orders. A critical care nurse collected demographic data, diagnosis at the time of admission, probability of survival, severity of illness, resource consumption, nursing care requirements and in-hospital outcome. Patients classified as DNR tended to be older, Caucasian, have poor health status, higher admission severity of illness, poorer prognosis for survival and longer stays in intensive care. Patients classified as DNR received higher levels of nursing care based on the patient care categorization tool used at the hospital than all the other patients. Intense levels of nursing care were required after the DNR order.

\section{Method}

The present research was a prospective study. Patients in intensive care were classified as DNR or non-DNR. Data collected for all patients included nursing care requirements, severity of illness, resource allocation and socio-demographic characteristics.

\section{Sample}

The sample consisted of 62 DNR patients and 62 nonDNR patients in the intensive care units of three community hospitals. All patients who had a DNR order during their stay in intensive care were admitted into the study. When a DNR patient was admitted into the study, a random non-DNR patient in the same ICU was admitted in the study. Room numbers in the ICU's were used to identify the patients and a table of random numbers was used to select the non-DNR patients. Patients with DNR orders were not matched with non-DNR patients for characteristics. Patients in intensive care for overnight monitoring after surgery or to rule out a myocardial infarction were excluded from the study. Patients in the ICU for open heart surgery were also excluded due to differences in the severity of illness scores of these patients as compared to other patients in intensive care (Zimmerman et al., 1986). Patients in both groups had to be in intensive care for a minimum of 24 hours to be admitted to the study.

\section{Instruments}

The Nursing Productivity and Quality System (NPAQ) (Medicus Systems Corporation, 1985) was used to measure nursing care requirements. The NPAQsystem was developed by the Medicus Systems Corporation to classify the acuity of patients in order to determine nursing staffing patterns for hospitals. The patient's nursing needs are reflected by the acuity of the patients. Patients with greater needs will require more nursing care. The NPAQ System consists of 37 indicators that reflect nursing care needs of patients, including physical, psychosocial and emotional needs. The indicators are weighted and the patients are classified as Type I - V. Validity of the system has been estimated by Medicus Systems Corporation (Medicus Systems Corporation, 1985). The five-type classification tool was used in several hospitals' intensive care units. Overall agreement between estimated and calculated patient type was 89 percent with a range of 86-95 percent (Medicus Systems Corporation, 1985). Calculated patient type was derived from the five-type tool and estimated patient type was derived from an assessment of the patient by the nurse as to hours of nursing care required in 24 hours. 
The APACHE II (Acute Physiology and Chronic Health Evaluation) severity of disease classification system (Knaus, Draper, Wagner, \& Zimmerman, 1985) was used to measure severity of illness. APACHE II is composed of a score on 12 routine physiologic measurements, along with the patient's age and chronic health status. The physiologic variables plus points for age and chronic health status are weighted and summed for one score. The maximum possible score is 71 . Past reliability and validity studies have been estimated through comparison of APACHE II scores and mortality rates. Knaus et al. (1985) contend that the most specific standard for determining the validity of severity of disease is mortality. Observed and predicted death rates were compared in several studies (Knaus et al., 1985; Knaus, Draper, Wagner, \& Zimmerman, 1986; Wagner, Knaus, \& Draper, 1986). Significantly higher death rates were seen with higher APACHE II scores. In addition, Knaus et al. (1985) compared the APACHE II System with the original APACHE for accuracy. The percent of cases correctly classified was 85 for both instruments and the correlation between outcome and predicted probability was .73 for both instruments. Interrater reliability has been estimated on the 12 physiologic parameters of the APACHE II with a 96 percent agreement between data collectors (Wagner et al., 1983, 1986).

The Therapeutic Intervention Scoring System (TISS) (Keene \& Cullen, 1983) was used to measure resource consumption. Severity of illness can be classified using TISS by counting and weighting the therapeutic interventions used by patients on the assumption that one more critically ill patient usually requires more therapeutic interventions. The TISS consists of 76 therapeutic interventions scored on a on-to-four point scale. The interventions are summed for the previous 24 hours. Examples of the therapeutic interventions include controlled ventilation, hyperalimentation, chest tubes and peritoneal dialysis. Construct validity has been estimated by correlating the TISS and APACHE II and the probability of death (Knaus, Zimmerman, Wagner, Draper, \& Lawrence, 1981; Scheffler, Knaus, Wagner, \& Zimmerman, 1982). Estimates of validity were found to be acceptable $(\underline{r}=.51-.59, \underline{p}<.01)$.

The sociodemographic characteristics measured for this study included: (a) age; (b) gender; (c) ethnic group; (d) place of residence; (e) marital status; (f) length of intensive care stay; and ( $\mathrm{g}$ ) origin of admission. The origin of admission included: operating room (OR); recovery room (RR); emergency room (ER); a general nursing unit (GNU); and others, such as another hospital. Origin of admission to intensive care has not been previously studied.

Construct validity was estimated on APACHE II, TISS and NPAQ by collecting data on a sample of 15 patients from a general medical/surgical unit and comparing the data to a separate sample of 15 patients admitted to intensive care in one hospital. These two groups would be expected to differ on severity of illness, resource allocation and nursing care requirements. Admission data on the two groups were collected and compared using t-tests for independent samples. The t-test results on all three instruments were significantly different.

As APACHE II and TISS reflect measures of severity of illness, construct validity was estimated by correlating the two instruments. Correlations of the same or related measures are an acceptable method for construct validity (Waltz,
Strickland, \& Lenz, 1984). A Pearson Product Moment Correlation Coefficient was determined $(\underline{\mathrm{r}}=.72 ; \mathrm{p}<.01)$ and provided evidence of construct validity.

\section{Data Collection}

Permission was obtained from each of the three participating institutions. Patients in the ICU with DNR orders were compared with patients in the ICU who were to be resuscitated. Some patients in the non-DNR group received DNR orders during their intensive care stay and were then admitted to the DNR group. Data collection was on a daily basis through chart reviews, observation of the patient and discussions with the nursing staff. All data were collected by the principal investigator until subjects recovered and were transferred from the unit or until death occurred.

\section{Results}

The sample consisted of 62 DNR and 62 non-DNR patients. The sociodemographic characteristics of the sample are summarized in Table 1.

The mean age of the DNR patients was 74 years and the mean of the non-DNR patients was 67.7 years. Most of the DNR patients (79 percent) and non-DNR (92 percent patients) lived in their own home or with family. For DNR patients, 21 percent resided in a nursing home, while only eight percent of non-DNR patients resided in a nursing. The majority of the patients in both groups, approximately half the patients were male and half were female. The majority of patients in both groups were married.

Logistic regression were used to determine which variables were most predictive of a DNR classification. Origin of admission to the ICU, severity of illness score on the day of admission to the ICU and demographic variables were among those considered for inclusion in the model. Severity of illness score on the day of admission to the ICU and origin of admission were found to be associated significantly with the odds of being DNR. No other variables were statistically significant once these two variables were in the model. We also considered models that controlled for differences in

\begin{tabular}{lcc}
\hline $\begin{array}{c}\text { Table 1. Sociodemographic Characterisitics of the } \\
\text { Sample }\end{array}$ & $\begin{array}{c}\text { Non-DNR Patients } \\
(\mathrm{n}=62)\end{array}$ \\
\hline & $\begin{array}{c}\text { DNR Patients } \\
(\mathrm{n}=62)\end{array}$ & 67.7 \\
\hline Age (mean yr) & 74 & \\
Residence (\%) & & 8 \\
Nursing Home & 21 & 92 \\
$\quad$ Home/Family & 79 & \\
Ethnic Group (\%) & & 84 \\
White & 97 & 16 \\
Non-white & 3 & \\
Gender (\%) & & 53 \\
Male & 47 & 46 \\
Female & 54 & \\
Marital Status (\%) & & 58 \\
Married & 42 & 5 \\
Divorced & 8 & 25 \\
Widowed & 36 & 0 \\
Separated & 2 & 13 \\
Single & 8 & \\
\hline
\end{tabular}


Table 2. Estimated Relative Odds of a DNR Classification for Patients in Intensive Care

\begin{tabular}{lcc}
\hline Origin of Admission & Estimate & $95 \%$ Confidence Interval \\
\hline Operating Room & 1.00 & baseline category \\
Recovery Room & 0.30 & $(0.09,1.00)$ \\
Emergency Room & 0.38 & $(0.15,0.95)$ \\
General Nursing Unit & 1.78 & $(0.66,4.85)$ \\
Other & 0.04 & $(0.01,0.19)$ \\
\hline
\end{tabular}

NOTE: Odds caclulated by logistic regression

demographic variables, but found that controlling for such differences did not alter our conclusions. In other words, none of the demographic variables were found to be statistically significant predictors of a DNR classification.

It was not possible to directly estimate the probability of a DNR classification from our study design. Nonetheless, it is possible to estimate the relative odds of a DNR classification (Breslow \& Day, 1980). The interpretation of estimated effects is in terms of the relative odds of a DNR classification, where the odds of a DNR classification is the probability of being DNR, divided by the probability of not being DNR. An increase in odds then corresponds to an increase in the probability of being DNR. The interpretation of estimates given in Table 2 is in terms of the relative odds of a DNR classification, controlling for severity of illness. The relative odds of a DNR classification is the origin of admission relative to the operating room. For example, the odds of a DNR classification for patients admitted from the recovery room are 0.30 times the odds for patients admitted from the OR, with $(0.09,1.00)$ being the 95 percent confidence interval for the relative odds. Therefore, patients admitted from the OR appear more likely to be classified DNR than patients admitted from the $\mathrm{RR}$, but the evidence is relatively weak, since the confidence interval does include 1.00.

To determine the best predictors of nursing care requirements of DNR patients in intensive care, resource allocation, severity of illness and length of stay in intensive care were examined using linear regression analysis. The simplest model that accurately described nursing care requirements is given as:

$$
\begin{aligned}
& \mathbf{E}(\mathbf{Y})=\alpha+\beta 1 \mathrm{X}_{1}+\beta 2 \mathrm{X}_{2}+\beta 3 \mathrm{X}_{3} \text { where } \\
& \mathrm{Y} \text { is average daily nursing care points after the DNR }
\end{aligned}
$$

No other variables, including demographics, were statistically significant once the three variables given above were included in the model. Table 3 summarizes the variables used in the model and the parameter estimates. The $\underline{\mathrm{R}} 2$ for this model was 63 percent and all the models for nursing care requirements considered, this one provided the best summary of the data.
Table 3. Parameter Estimates for the Model to Predict Nursing Care of Patients in Intensive Care with DNR Orders

\begin{tabular}{lcl}
\hline Parameter & Estimate & Std. Error \\
\hline$\alpha$ & 83.73 & 8.31 \\
$\beta 1$ & 0.97 & 0.27 \\
$\beta 2$ & 1.76 & 0.32 \\
$\beta 3$ & 1.02 & 0.28 \\
\hline & & \\
$R^{2}=63 \%$ & \\
$S=16.73$ (estimate standard deviation) \\
\hline
\end{tabular}

\section{Discussion}

The DNR and non-DNR patients were not matched for demographic characteristics and non-probability methods were used to select the DNR subjects. This raises the possibility that selection bias may have been introduced. However, regression models that controlled for demographic differences found none of these to be statistically significant. Observed differences between the groups did not seem to be the result of demographic differences. Thus, comparison of the two groups appears to be valid. In addition, non-DNR patients in intensive care with certain medical diagnoses were excluded from the study, i.e., patients in intensive care for overnight monitoring, to rule out myocardial infarction and for open heart surgery. Due to the short intensive care stay and the low severity of illness, patients in intensive care for overnight monitoring and to rule out myocardial infarction would probably not receive DNR orders. The severity of illness scores of patients having open heart surgery are so different from other patients in intensive care, they were excluded from the study (Zimmerman, et al. 1986).

Few patients in either group were non-white. The most current life expectancy tables (Florida Department of Health and Rehabilitative Services, 1990) show that years of life expectancy for Florida residents by race and sex are notably discrepant: white males-71.1; white females-78.9; non-white males-63.8; and non-white females-72.4. Although we cannot use these data to explain why more non-whites were not in the sample, it is postulated that non-whites are less likely to be admitted to the study hospitals because they do not live in the service area; they have fewer medical resources such as comprehensive health insurance coverage; and, nonwhites are more likely to die at home. Further research is needed to examine the occurrence of DNR status by race and sex.

Other studies have examined demographic characteristics to determine which were the best predictors of a DNR designation (Bedell, Pelle, Maher, \& Cleary, 1986; Uhlmann, McDonald, \& Inui, 1984). In the present study, results indicate that important predictors of a DNR order for patients in ICU are the origin of admission for the subject and the admission severity of illness score. Severity of illness was most strongly associated with a DNR classification of patients in intensive care in the present study. Origin of admission, though statistically significant, was only weakly associated with DNR classification. The two studies examining the best predictors of a DNR classification for subjects in 
intensive care (Younger et al., 1985; Zimmerman et al., 1986) also found severity of illness to be a predictor of a DNR designation of patients in intensive care.

No other sociodemographic characteristics or variables were significant predictors of a DNR classification in the present study. This finding is different from other studies where age and prior health status were also significant predictors (Younger et al., 1985; Zimmerman et al., 1986). However, age and prior health status are used in computing the APACHE II score. Even though age and prior health status were found not to be individually predictive in the present study, they are relevant through the APACHE II system.

In reviewing the relative odds of receiving a DNR classification, the odds were lowest for patients in the "other" category of admission origin. Five patients were in this category, four who did not have DNR orders. The patient with the DNR order was admitted to the ICU from a rehabilitation unit. One of the non-DNR patients was transferred to intensive care from another hospital. The remaining nonDNR patients were direct admissions to the ICU from physicians' offices. When such admissions occur, often the physician has advised the patient to go directly to the hospital. Patients of this type do not go to the emergency room but to the admitting office and are considered direct admissions. Patients in the "other" category may have only required monitoring to prevent complications and were not severely ill.

The model predicting nursing care of DNR patients includes three variables: (a) the number of days the subject was in intensive care prior to the DNR order; (b) the average daily resource allocation points after the DNR order; and (c) the severity of illness score on the day of the DNR order. Lewandowski et al. (1985) examined the nursing care of DNR patients in intensive care but did not develop predictive models. No other studies were found to predict nursing care of DNR patients in intensive care.

Two outliers or influential observations were present in this model - two patients who were in intensive care for 35 days and 45 days prior to the DNR order. The estimate of $\beta 1$ for the variable of number of days in intensive care prior to the DNR order increased from 0.97 to 2.03 without these two patients in the data set. For DNR patients with long intensive care stays, the nursing care required may level off and remain unchanged. As a result, it appears that a different model for nursing care needs would be appropriate for longterm intensive care admissions.

The present study is a preliminary investigation for predictive models. Further validation of the models is required by additional research on the DNR patient. The DNR patient continues to require labor-intensive nursing care and therapeutic interventions even though the DNR order implies the patient is hopelessly ill. Clearly, DNR orders do not imply abandonment of the patient. Do-not-resuscitate decisions should be separate from treatment decisions. However, discussion with patients and families should include whether certain treatments will have a positive benefit or prolong death unnecessarily. Nurses provide the bulk of patient care and have the best insight as to the wishes of the patient and family. The continued development of predictive models for nursing care will help provide objective data for decisions regarding care needs for these patients.
Further research is necessary to determine predictors of a DNR designation of patients in intensive care as well as the nursing care required. The predictors of a DNR classification could then be used to make decisions regarding appropriate health care based on prognosis. In view of the ethical consideration of DNR decisions, it is unlikely that specific guidelines for reducing or withdrawing therapy will be developed. However, continued research on characteristics of patients with a DNR status will serve to provide objective information that will assist health care professionals to provide care to meet the needs of these patients.

\section{References}

Bedell, S.E., Pelle, D., Maher, P.L., \& Cleary, P.D. (1986). Do-not-resuscitate orders for critically ill patients in the hospital. How are they used and what is their impact? Journal of the American Medical Association, 256, 233-237.

Breslow, N.E. \& Day, N.E. (1980). Statistical methods in cancer research. Lyon, FR: International Agency for Research on Cancer.

Cullen, D.J., Keene, R., Waternaux, C., Kunsman, J.M., Caldera, D.L., \& Peterson, H. (1984). Results, charges and benefits of intensive care for critically ill patients. Update 1983. Critical Care Medicine, 12, 102-106.

Draper, E.A. (1983). Benefits and costs of intensive care. IMAGE: The Journal of Nursing Scholarship, 15, 90-94.

Florida Department of Health and Rehabilitative Services. (1990). Vital News, 3(3), 9-11.

Jackson, B.S. (1984). A one-year mortality study of the most acutely ill patients in a medical-surgical intensive care unit: Toward developing a model for selection of recipients of intensive care. Heart \& Lung, 13, 132-137.

Keene, A.R. \& Cullen, D.J. (1983). Therapeutic intervention scoring system: Update 1983. Critical Care Medicine, 11, 1-3.

Knaus, W.A., Draper, E.A., Wagner, D.P., \& Zimmerman, J.E. (1985), APACHE II: A severity of disease classification. Critical Care Medicine, 13, 818-829.

Knaus, W.A., Draper, E.A., Wagner, D.P., \& Zimmerman, J.E. (1986). An evaluation of outcome from intensive care in major medical centers. Annals of Internal Medicine, 104, 410-418.

Knaus, W.A., Wagner, D.P., Draper, E.A., Lawrence, D.E., \& Zimmerman, J.E. (1981). The range of intensive care services today. Journal of the American Medical Association, 246, 2711-2716.

Knaus, W.A., Zimmerman, J.E. Wagner, D.P., Draper, E.A., \& Lawrence, D.E. (1981). APACHE - Acute Physiology And Chronic Health Evaluation: A Physiologically based classification system. Critical Care Medicine, 9, 591-597.

Kouwenhoven, W.B., Jude, J.R., \& Knickerbocker, G.G. (1960). Closed-chest cardiac massage. Journal of the American Medical Association, 173, 1064-1067.

Lewandowski, W., Daly, B., McClish, D.K. Juknialis, B.W., \& Younger, S.J. (1985). Treatment and care of "do not resuscitate" patients in a medical intensive care unit. Heart \& Lung, 14, 175-181.

Medicus Systems Corporation. (1985). Fifth patient type classification tool. Overview of the validation process. Evanston, IL. The Corporation.

Parno,J.R., Teres, D., Lemeshow, S., \& Brown, R.B. (1982). Hospital charges and long term survival of ICU versus non-ICU patients. Critical Care Medicine, 10, 567-574.

Scheffler, R.M., Knaus, W.A., Wagner, D.P., \& Zimmerman, J.E. (1982). Severity of illness and the relationship between intensive care and survival. American Journal of Public Health, 72, 449-454.

Thibault, G.E., Mulley, A.G., Barnett, G.O., Goldstein, R.L., Reder, V.A., Sherman, E.L., \& Skinner, E.R. (1980). Medical intensive care: Indications, interventions, and outcomes. The New England Journal of Medicine, 302, 938-942.

Uhlmann, R.F., McDonald, W.J., \& Inui, T.J. (1984). Epidemiology of no-code orders in an academic hospital. Western Journal of Medicine, 140, 114-116.

Wagner, D.P., Knaus, W.A., \& Draper, E.A. (1986). Physiologic abnormalities and outcome from acute disease. Archives of Internal Medicine, 146, 1389-1396.

Wagner, D.P., Knaus, W.A. Draper, E.A., \& Zimmerman, J.E. (1983). Identifications of low risk monitor patients within a medical-surgical intensive care unit. Medical Care, 21, 425-434.

Waltz, C.F., Strickland, O.L., \& Lenz, E.R. (1984). Measurement in nursing research. Philadelphia: F.A. Davis Company.

Witte, KL. (1984). Variables present in patients who are either resuscitated or not resuscitated in a medical intensive care unit. Heart \& Lung, 13, 159-163.

Younger, S.J., Lewandowski, W., McClish, D.K, Juknialis, B.W., Coulton, C., \& Bartlett, E.T. (1985). "Do not resuscitate" orders. Incidence and implications in a medical intensive care unit. Journal of the American Medical Association, 253, 5457.

Zimmerman, J.E., Knaus, W.A., Sharpe, S.M., Anderson, A.S., Draper, E.A., \& Wagner, 